\title{
Perioperative Anaesthesia Management of Patient with Dilated Cardiomyopathy Posted for Total Abdominal Hysterectomy
}

\author{
Dr. Asegaonkar Balaji N \\ M.D. D.N.B. Cardiac Anaesthesiology, Consultant Anaesthesiologist \\ Kodlikeri Memorial Hospital \& CIIGMA Hospital \\ Aurangabad, Maharashtra India. \\ b_asegaonkar@yahoo.com \\ Dr. Preeti Bhagyawant D.A \\ Anesthesiologist Kodlikeri Memorial Hospital \& CIIGMA Hospital \\ Aurangabad, Maharashtra India \\ Dr. Pushpa Kodlikeri M.B.B.S., MIFEE, MIAHR \\ Gynaecologist, Infertility Specialist \& Laparoscopist, Medical Director \\ Kodlikeri Memorial Hospital \& CIIGMA Hospital, Aurangabad, India \\ pushpa.kodlikeri@gmail.com \\ Dr. Unmesh Takalkar M.S. (Gen.Surg.) \\ M.E.D.S., F.U.I.C.C. (Switzerland), MSSAT (USA), \\ Cancer General \& Endoscopic Surgeon, Observer, Johns Hopkins (USA) Consultant Surgeon and \\ Medical Director Kodlikeri Memorial Hospital \& CIIGMA Hospital \\ Aurangabad, India \\ drunmesh.aurangabad@gmail.com
}

\begin{abstract}
Dilated cardiomyopathy (DCM) is characterized by progressive cardiac dilation resulting in impaired systolic function of one or both ventricles. Perioperative anaesthetic management of patients with DCM undergoing noncardiac surgery always poses several challenges to anaesthesiologist. Progressive cardiac failure and malignant arrhythmias worsen outcome in such patients.

We share our experience of anaesthesia considerations while managing obese patient with severe DCM and co morbidities of hypertension and hypothyroidism. We managed present case with controlled epidural anaesthesia, which offered excellent hemodynamic stability. Another distinct advantage of this technique is good quality postoperative analgesia throughout perioperative period.
\end{abstract}

Keywords: Dilated cardiomyopathy, anaesthetic management, epidural anaesthesia.

Keymessage: Patients with DCM require careful perioperative anaesthetic management for noncardiac surgery. Common perioperative complications are acute left ventricular failure and arrhythmias, which can affect perioperative outcome in these patients.

\section{INTRODUCTION}

Dilated cardiomyopathy (DCM) is characterized by progressive cardiac dilation resulting in impaired systolic function of one or both ventricles. ${ }^{[1]}$ Its prevalence is 36 per 100000 with dominance in males. Although idiopathic, familial association is observed in case of DCM. It may be of ischemic type related to atherosclerosis and nonischemic variety may be secondary to viral infections, alcoholism, hypothyroidism or chemotherapy induced. DCM can also be associated with sickle cell anemia, valve dysfunction, and muscle dystrophy and during peripartum period. ${ }^{[2,3]}$

Perioperative anaesthetic management of patients with DCM undergoing noncardiac surgery always poses several challenges to anaesthesiologist. Progressive cardiac failure and malignant arrhythmias worsen outcome in such patients. We share our experience of perioperative management of obese patient with severe DCM and co morbidities of hypertension and hypothyroidism. 


\section{Case Presentation}

A 47 year old, $70 \mathrm{~kg}$ lady was admitted to our institute with dysfunctional uterine bleeding due to fibroid. After thorough clinical evaluation and diagnostic work up, she was posted for abdominal hysterectomy. She was obese with body mass index $29 \mathrm{~kg} / \mathrm{m}^{2}$ and known case of hypertension since 7 years, hypothyroidism and Ischemic Heart Disease (IHD) since 5 years. She was also diagnosed to have DCM when she was evaluated for IHD. Her coronary angiography was normal. Her comorbidites were managed with regular tablet Eltroxin 25ug, Lanoxin $0.25 \mathrm{mg}$, Metaprolol $25 \mathrm{mg}$ once a day and Ramipril $2.5 \mathrm{mg}$ twice daily.

Pre-anaesthetic evaluation revealed regular heart rate of $64 / \mathrm{min}$, a blood pressure of $110 / 70 \mathrm{mmHg}$, $\mathrm{SpO}_{2} 94 \%$ on room air. General examination showed pallor ++ and minimal oedema over feet. Clinically she had poor effort tolerance with grade III dyspnea (NYHA classification). There was no history of orthopnea or paroxysmal nocturnal dyspnea.

Chest $\mathrm{X}$ ray showed cardiomegaly with pulmonary congestion. On auscultation occasional crepts and grade III systolic murmur was present. Electrocardiogram revealed left bundle branch block. Echocardiography examination showed dilated left ventricle (LV) with severely depressed systolic function (Ejection fraction 25\%). Also patient had sclerotic valve with mild aortic regurgitation. In context of cardiological evaluation, cardiologist insisted aggressive antifailure treatment. Injectable Torsemide $20 \mathrm{mg}$ IV and syrup Kesol 2 table spoon twice daily administered 2 days prior to surgery. With this management she had symptomatic relief with SpO2 99\% without crepts on auscultation.

Airway assessment showed Mallampati grade II difficult intubation. Routine blood and urine investigations, serum electrolytes, liver and renal function tests were within normal limit. Serum T3 and T4 levels were within normal range while there was borderline rise in TSH level to $5.6 \mathrm{~m} \mathrm{IU} / \mathrm{ml}$. She was HBsAg positive.

High risk was explained to patient and relatives and informed written consent was obtained. Her physical status was ASAIIIclass. On the pre-operative night, tablet Alprazolam $0.5 \mathrm{mg}$ HS and capsule Omez $20 \mathrm{mg}$ were advised. Patient was kept nil by mouth. On the day of surgery, tablet Eltroxin $0.25 \mathrm{mg}$, metaprolol $25 \mathrm{mg}$ were administered. In the operating room monitoring started with five lead ECG, pulse oxymetry, NIBP and baseline vital parameters were recorded. Under strict aseptic precautions, right jugular vein cannulated with triple lumen central venous pressure (CVP) catheter. Her baseline CVP was $9 \mathrm{mmHg}$ and it was continuously monitored.

In sitting position $18 \mathrm{G} \mathrm{B}$ Braun epidural catheter was inserted at L1-2 up to $4 \mathrm{~cm}$ into epidural space through Touhy needle in cephalad direction by normal saline technique. Epidural catheter was fixed properly and $3 \mathrm{ml}$ test dose of Xylocaine $2 \%$ with Adrenalin was given to confirm proper placement. Patient was monitored for vital signs for three minutes to rule out intravenous placement. After 10 minutes, $8 \mathrm{ml}$ of $0.75 \%$ Ropivacaine and $1 \mathrm{ml}$ Fentanyl (50 microgram) injected through catheter. This resulted in bilateral anaesthesia in the area up to T5/6 level in next 20 minutes. After achieving dense sensory block and analgesia patient was handed over to surgeon for abdominal hysterectomy. Patient was haemodynamically stable during intraoperative phase. There was fall in blood pressure by $20 \%$ which was managed by infusion of 1 Lt Fluid (500 $\mathrm{ml} \mathrm{RL}$ and $500 \mathrm{ml}$ Voluven). Patient was sedated with Fentanyl 50 microgram and midazolam 1mg. To avoid arrhythmias and cardiac irritability, $20 \mathrm{mEqKesol}$ and $1 \mathrm{gm}$ Magnesium was administered over 1 hour. Surgery lasted for 60 minutes without any adverse event.

Patient shifted to postoperative anaesthesia care unit for further management. As per institutional protocol analgesia was provided with injection Bupivacaine $0.125 \%$ and Fentanyl 10 ug every 4-6 hourly bolus through epidural catheter. Hemodynamic and biochemical parameters were within stipulated limits throughout immediate postoperative period. Also she was comfortable and pain free. Next day she was shifted to ward and discharged from hospital on $6^{\text {th }}$ day.

\section{DisCUSSION}

DCM, a primary myocardial disease of varied aetiology is defined by a large heart cavity with impaired systolic function of one or both ventricles. It is characterized by presence of fractional myocardial shortening $<25 \%$ or ejection fraction $<45 \%$ and left ventricular end diastolic diameter $>$ $117 \%$ in absence of other known causes of myocardial diseases. Clinically patient presents with dyspnea, orthopnea weakness, fatigue, ascites and pedal oedema. Systemic examination findings are 
similar to that of congestive cardiac failure like elevated jugular venous distension, rales, pulmonaryoedema, resting tachycardia, S3 and S4 heart sound and cardiomegaly. ${ }^{[4]}$ Before posting such patient for any surgery, it is advisable to decongest them with diuretics. This will improve perioperative hemodynamic.

In our case patient had associated co morbid conditions of hypothyroidism and hypertension. In preoperative check up, she was found to be HBsAg positive. Our target for managing this case was to optimize her ventricular systolic function and prevent arrhythmias throughout perioperative period. While central venous cannulation, utmost care were taken especially with guide wire to avoid arrhythmias. There are many reports of Guide wire induced serious arrhythmias in literature. ${ }^{[5]}$

Anaesthesia management in patients with DCM is challenging one. Its detection in preoperative evaluation is important as $10.1 \%$ of 1100 patients had been found to have DCM. ${ }^{[6]}$ Chang KH reported major complications in six and minor in 23 of 73 cases of DCMP patients (Mean EF- 31\%) undergoing noncardiac surgery. ${ }^{[7]}$ Also he commented lack of preoperative diagnosis of DCM and $\mathrm{CHF}$ are significant risk factors. Evaluation of cardiac reserve is more important than the resting value of EF. Bansal T. et al used spinal anaesthesia for their patient with DCM (EF- 35\%) planned for fracture of trochanter. They managed frequent episodes of ventricular ectopic with injection xylocard infusion. ${ }^{[3]}$ Surbhi et al reported splenectomy under general anaesthesia in a patient with severely DCM. ${ }^{[8]}$

Preoperative evaluation aims at optimization of cardiac failure medically by antifailure medication prior to surgery. Our patient had other co morbidities like hypertension which were well controlled. As far as anaesthesia management is concerned, target is to provide stable hemodynamic throughout perioperative period. Rather than technique, one should aim to minimize alteration in the hemodynamic state. ${ }^{[9]}$ In this regard, first choice was general anaesthesia, but it itself was associated with intubation and extubation responses. Another choice was regional anaesthesia which could have produced minimal hemodynamic alterations. So we decided to go ahead with segmental epidural anaesthesia.

Overall aim of anaesthesia was to avoid tachycardia, negative inotropism, and sudden increase in after load and maintain adequate preload. This was achieved by avoiding administration of various drugs for GA. We put CVP catheter to monitor preload and for aggressive hemodynamic monitoring. Another important issue in management is to avoid arrhythmias and maintain sinus rhythm. For that we continued patient on beta blocker and serum potassium maintained at $4.5 \mathrm{mEq} / \mathrm{L}$ to avoid irritability of heart. Sudden arrhythmias can have worst prognosis in such cases. Another major advantage of epidural was good quality pain management with repeated epidural analgesic dosages which avoided sudden rise in systemic vascular resistance and heart rate.

Thorough cardiological examination and treatment from cardiologist prior to surgery result in better outcome. We successfully anesthetize the patient of severe DCMP with co morbidities by choosing option of epidural anaesthesia. Present case report provides an insight to approach a patient with severely DCM with hypothyroidism and hypertension posted for noncardiac surgery.

\section{CONCLUSION}

Compared to GA, controlled epidural anaesthesia offers excellent hemodynamic stability in case of dilated cardiomyopathy patient. Another distinct advantage of this technique is good quality postoperative analgesia throughout perioperative period.

\section{REFERENCES}

[1] Dec GW, Foster V. Idiopathic dilated cardiomyopathy. N Eng J Med 1994;33:1564-75

[2] Mr Davies BM, J Cousins. Cardiomyopathy and anaesthesia. Continuing Education in Anaesthesia, Critical Care \& Pain 2009;9(6): 189-93.

[3] Bansal T, Hooda S. Anaesthetic Management of an Elderly Patient with Dilated Cardiom yopathy Undergoing Surgery for Fracture of Trochanter. WebmedCentral ANAESTHESIA 2011; 2(12):WMC002567.

[4] Prashan H. Thiagarajah, SomasundaramThiagarajah and Elizabeth A.M. Frost. Anesthetic Considerations in Patients with Cardiomyopathies M.E.J. ANESTH 2009;20 (3) 347-54. 
[5] Stuart RK, Shikora SA, Akerman P, Lowell JA, Baxter JK, Apovian C, Champagne C, Jennings A, Keane-Ellison M, Bistrian BR. Incidence of arrhythmia with central venous catheter insertion and exchange. JPEN J Parenter Enteral Nutr. 1990 Mar-Apr;14(2):152-5.

[6] SangeetNarang. Anesthetic Management of Dilated Cardiomyopathy. Middle East $\mathrm{J}$ of Anesthesia 2007;19(1):243-50.

[7] Chang KH, Hanoka K. Anesthetic management of patients with dilated cardiomyopathy undergoing non-cardiac surgery. Masui 2004;53(12):1360-8.

[8] Surbhi D Mundada, Bharat Shah. Anesthetic management of a case of severe Dilated Cardiomyopathy with Splenic Abscess for Splenectomy: Case report. The Indian Anesthetist's Forum July 2011;1-4.

[9] Mohamed A. Daabiss, Adel Hasanin. Perioperative Anesthetic Management of a Case with Severe Dilated Cardiomyopathy Oman Medical Journal 2010;25(1). 